\title{
Minibands and Wannier-Stark Ladders in Semiconductor Superlattices Studied by Infrared Spectroscopy
}

\author{
M. Helm, W. Hilber, \\ Institut für Halbleiter- und Festkörperphysik, Universität Linz, A-4040 Linz, Austria \\ G. Strasser, \\ Institut für Festkörperelektronik, TU Wien, A-1040 Wien, Austria \\ R. DeMeester, and F. M. Peeters \\ Department of Physics, University of Antwerp (UIA), B-2610 Wilrijk, Belgium
}

Received 27 May, 1999

\begin{abstract}
We review the basic features of interminiband absorption in superlattices, focussing on the joint density of states, the oscillator strength and the associated sum rule. Then we will discuss infrared spectroscopic studies under application of an electric field. With a field in the plane of the layers, a hot-electron distribution can be generated. Using the temperature dependence of the interminiband absorption, energy loss and relaxation rates can be determined. A vertical electric field leads to negative differential resistance, domain formation and the break-up of the minibands into WannierStark ladders. We will present experiments showing the formation of interpenetrating WannierStark ladders in the continuum, which are strongly coupled by Zener resonances. This is the first time to correlate transport and infrared absorption in a biased superlattice.
\end{abstract}

\section{Introduction}

Semiconductor superlattices are a fascinating model system for electrons in a periodic potential [1]. They have enabled researchers to observe long-sought physical phenomena such as Wannier-Stark localization [2] and Bloch oscillations [3]. In the present paper we would like to review some of the basic properties of electrons in superlattices $\Gamma$ in particular those related to optical transitions between minibands within the conduction band $\Gamma$ which can be studied by infrared spectroscopy [4]. We will also present some recent absorption studies of superlattices under application of an electric field $\Gamma$ both parallel and perpendicular to the layers. Whereas in the former case information on the hot-electron relaxation is obtained [5] Tthe latter experiment reveals the formation of Wannier-Stark ladders far in the continuum and allows us $\Gamma$ for the first time $\Gamma$ to correlate the current-voltage characteristic with the infrared absorption spectrum [6].

\section{Interminiband absorption}

The conduction band of a GaAs/AlGaAs superlattice (SL) can be well described using the envelope function method $\Gamma$ which $\Gamma$ in the case of only one band $\Gamma$ reduces to the Kronig- Penney model with different effective masses in the well and barrier material. At first we would like to discuss a superlattice consisting of $75 \AA$ wide GaAs quantum wells and $25 \AA$ wide $\mathrm{Al}_{0.3} \mathrm{Ga}_{0.7} \mathrm{As}$ barriers. The calculated band structure is shown in Fig. 1. The first miniband has a width of $\Delta_{1}=18$ meVTthe second of $\Delta_{2}=70 \mathrm{meV}$ approximately. If the superlattice is doped with electronsToptical transitions can occur between all filled states of the first miniband and all empty states in the second miniband. It is well known that such transitions require the electric field of the exciting radiation to possess a non-vanishing component perpendicular to the SL layers (z-component) [7]. In quantum wells such so-called intersubband transitions have been extensively studied and have found applications in infrared detectors ("quantum well infrared 
photodetectors" TQWIPs) [8] and lasers ("quantum cascade lasers") [9]. In a strong- coupling superlattice $\Gamma$ as in the present case $\Gamma$ the thin barriers give rise to a fi- nite miniband width and $k_{z}$-dispersion. The absorption coefficient $\Gamma \alpha$ can then be written in the form [10]

$$
\alpha=\frac{e^{2} k T}{\epsilon_{0} c \eta \hbar^{2} \pi m^{*} \omega} \int_{0}^{\pi / d} d k_{z}\left|\left\langle 1\left|p_{z}\right| 2\right\rangle\right|^{2} \ln \left[\frac{1+\exp \left(\left[E_{F}-E_{1}\left(k_{z}\right)\right] / k T\right)}{1+\exp \left(\left[E_{F}-E_{2}\left(k_{z}\right)\right] / k T\right)}\right]\left(\frac{\Gamma / \pi}{\left(E_{2}\left(k_{z}\right)-E_{1}\left(k_{z}\right)-\hbar \omega\right)^{2}+\Gamma^{2}}\right)
$$

Here the parameters in the prefactor have their usual meaning $\Gamma \eta$ is the refractive index. The integration over $k_{x}$ and $k_{y}$ has already been performed assuming parabolic bands $\Gamma$ which leads to the logarithmic Fermi-Dirac factor. The energy-conserving $\delta$-function has been replaced by a Lorentzian with full-width at half-maximum (FWHM) of $2 \Gamma$. The integral over $k_{z}$ has to be evaluated numerically $\mathrm{T}$ using the miniband dispersions $E_{n}\left(k_{z}\right)$ in the Lorentzian. Note that also the matrix elements are $k_{z}$-dependent.

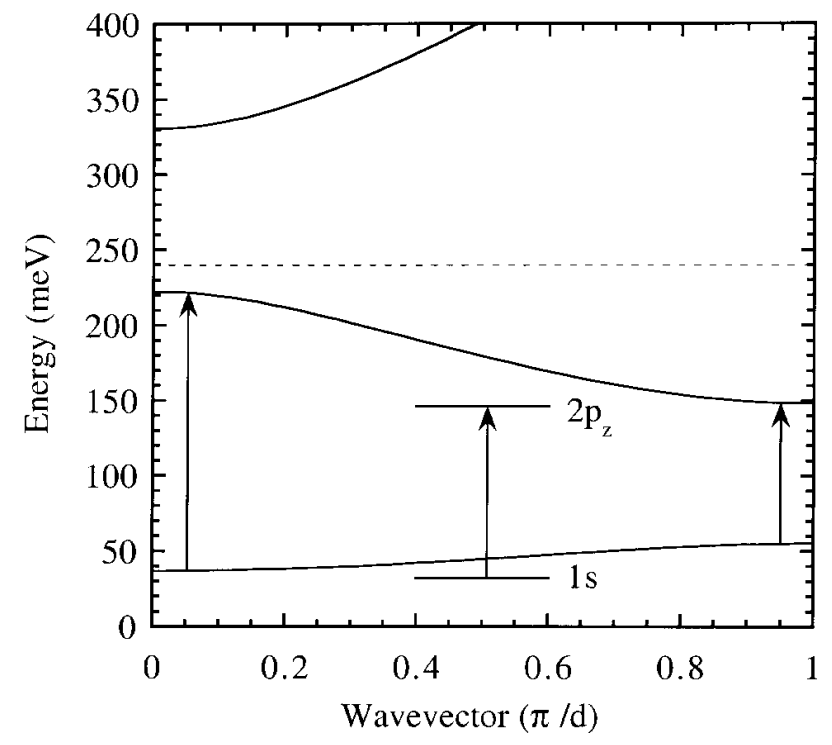

Figure 1. The calculated miniband structure of the $\mathrm{GaAs} / \mathrm{AlGaAs}$ superlattice under investigation (well width $75 \AA$, barrier width $25 \AA$ ). The horizontal dashed line indicates the top of the barriers. The $1 \mathrm{~s}$ and $2 p_{z}$ impurity states are also included schematically [4]. The interminiband transitions at the center and the edge of the mini-Brillouin zone are indicated as well as the impurity transition.

There are three essential contributions to the absorption coefficient: the squared momentum matrix el- ement (which is proportional to the oscillator strength through $\left.f_{12}=\left(2 / m^{*} h_{21}\right)\left|\left\langle 1\left|p_{z}\right| 2\right\rangle\right|^{2}\right) \Gamma$ the Fermi-Dirac thermal occupation factor $\Gamma$ and the Lorentzian. Together with the $k_{z}$ integration the latter is nothing less than the joint density of states (JDOS) Twhich has two singularities at the center $\left(k_{z}=0\right)$ and the edge $\left(k_{z}=\pi / d\right)$ of the mini- Brillouin zone with a $1 / \sqrt{\omega}$ divergence $\Gamma$ characteristic of its one-dimensional character. The JDOS for transitions between the two lowest minibands of the above superlattice is shown in Fig. 2 (dotted curve). The singularities are smoothed out by a broadening parameter of $\Gamma=10 \mathrm{meV}$. The full curve in Fig. 2 reflects the total absorption coefficient according to eq. 1 using an electron concentration of $6 \times 10^{17} \mathrm{~cm}^{-3}$ and a temperature of $T=5 \mathrm{~K}$. (At this doping level the Fermi energy lies above the top of the first minibandTi.e. T the first miniband is "full"). There is now a strong asymmetry with an enhanced low-frequency peak $\Gamma$ which corresponds to transitions at the edge of the mini-Brillouin zone (near $k_{z}=\pi / d$ ). The reason for this is the variation of the oscillator strength $\Gamma f_{12}\left(k_{z}\right) \Gamma$ across the Brillouin zone. In our present superlattice the oscillator strength varies from $f_{12}\left(k_{z}=0\right)=0.3$ to $f_{12}\left(k_{z}=\pi / d\right)=2.3$. This can be compared to the oscillator strength of an infinite single QWT which is $f_{12}=0.96$.

Such a behavior is characteristic of most superlattice band structures (it has actually been employed in miniband cascade lasers [11]) Tand is related to an extension of the well- known oscillator sum rule to periodic systems with energy bands. It reads

$$
\sum_{j} f_{i j}+\frac{m^{*}}{\hbar^{2}} \frac{\partial^{2} E_{i}\left(k_{z}\right)}{\partial k_{z}^{2}}=1
$$


or

$$
\sum_{j} f_{i j}\left(k_{z}\right)=1-\frac{m^{*}}{m_{S L}^{(i)}}
$$

$m_{S L}^{(i)}$ is the effective mass along the $z$-direction $\Gamma$ which is related to the curvature of the miniband at a certain point along $k_{z}$. In the above equation the first term describes transitions between different minibands (inter-miniband transitions). The second term $\Gamma$ which is absent in atomic system and contains the curvature of the miniband at a certain point along $k_{z}$ Tcorresponds to free-carrier type of transitions within one miniband [4]. From Eq. 2 it is clear that a large curvature (or a small effective miniband mass $m_{z}$ ) will strongly influence the values of $f_{i j}\left(k_{z}\right)$. Since the miniband curvature is positive near $k_{z}=0$ Tthe oscillator strength has to be reduced thereTwhereas it has to be enhanced due to the negative curvature near $k_{z}=\pi / d$ in order to fulfill the sum rule. Physically this corresponds to the possibility of free-carrier absorption and emissionTrespectively.

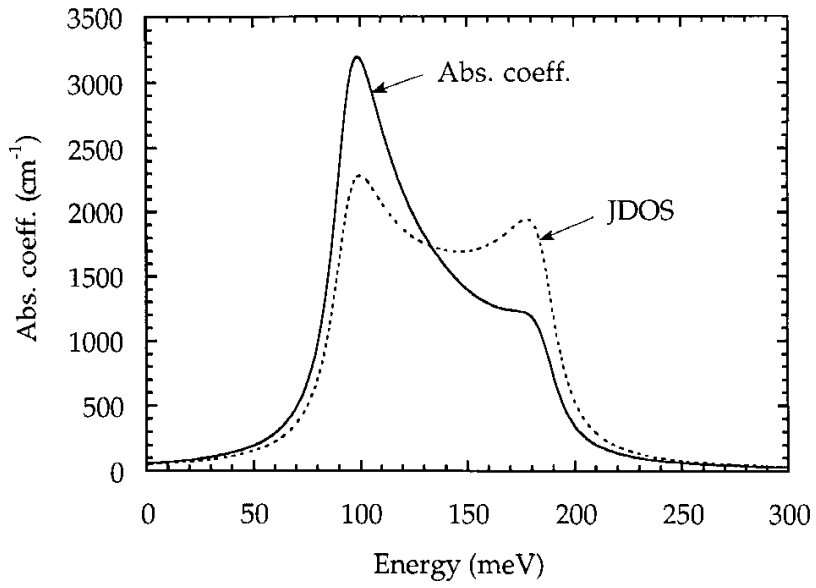

Figure 2. Calculated joint density of states (JDOS, dotted) and theoretical absorption coefficient for the above SL with $n=6 \times 10^{17} \mathrm{~cm}^{-3}$ at $\mathrm{T}=5 \mathrm{~K}$.

Let us now turn to the experiment. Two GaAs/AlGaAs SLs with the above mentioned parameters were investigated $\mathrm{T}$ one homogeneously doped to $n=6 \times 10^{17} \mathrm{~cm}^{-3}$ (having 200 periods) $\Gamma$ the other one to $n=6 \times 10^{16} \mathrm{~cm}^{-3}$ (and 500 periods). A crucial difference is that in the higher-doped sample the Fermi energy (at low temperature) lies above the top of the first miniband (which is "full") Twhereas in the lower-doped sample it lies approximately in the middle of the first miniband (it is "half-filled"). Both samples were prepared in a multi-pass waveguide geome- try with wedged facets in order to provide a finite $z$ component of the electric field of the infrared radiation and thus a coupling to the interminiband transition. The measurements were performed with a rapid-scan Fourier-transform spectrometer $\Gamma$ the samples mounted in a LHe flow-cryostat with variable temperature. The absorption spectrum of the higher-doped sampleTobtained by ratioing $\mathrm{p}^{-}$and s-polarized transmission $\Gamma$ is shown in Fig. 3. Evidently it agrees very well with the calculated spectrum in Fig. 2 and also does not change much with temperature. The situation is different for the lower-doped sample (Fig. 4). In this case the top-edge of the first miniband can be populated with electrons by increasing the temperature and thus the absorption spectrum becomes strongly temperature dependent. The temperature dependence is thus a clear proof of the $k_{z}$-dispersion of the first miniband. The additional line appearing at low temperature at $\hbar \omega=125$ meV is due to the $1 s-2 p_{z}$ donor transition [10] and its discussion is beyond the scope of the present paper [4].

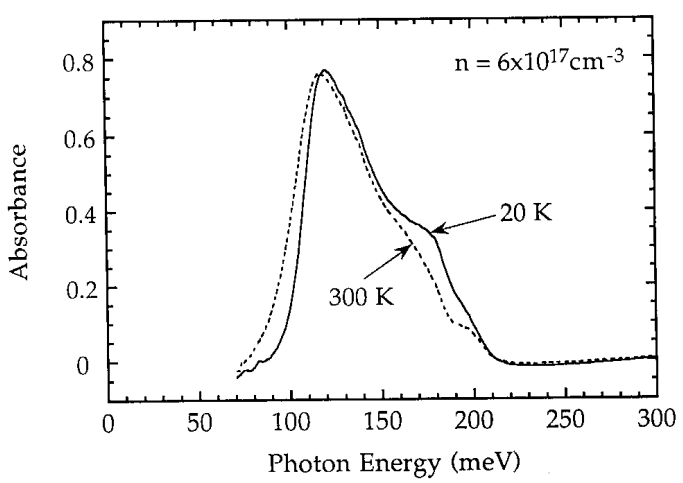

Figure 3. Measured absorption spectrum of the higher doped SL at $\mathrm{T}=20 \mathrm{~K}$ and $\mathrm{T}=300 \mathrm{~K}$ (absorbance $=-\log _{10}$ (transmission)). This should be compared with the theoretical curve in Fig. 2.

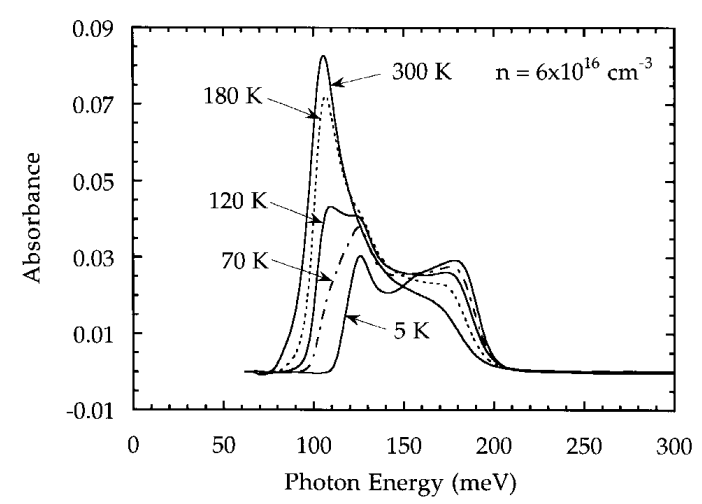

Figure 4. Measured absorption spectrum of the lower doped SL superlattice at different temperatures as indicated. For an explanation, see text. 


\section{Hot-electron relaxation}

In this section we would like to show Thow the temperature dependence of the interminiband absorption can be used as an intrinsic thermometer for the electrons $\Gamma$ and how the energy relaxation of the electrons can be measured [5]. When an electric field is applied parallel to the SL layers $\Gamma$ the electron distribution will be heated up. If impurity and electron- electron scattering are strong enough and the field is not too high Tthe resulting electron distribution will still be quasi-thermall with an electron temperature $T_{e}$. Since the shape of the absorption spectrum is mostly determined by the electron distribution $\Gamma$ the electron temperature can be deduced by comparing the spectra at certain electricfield values with spectra at certain lattice temperatures. FinallyIthe electron temperature reached in the electric field allows one to determine the electron heating and $\Gamma$ in steady state $\Gamma$ cooling rate.

For the experiment the above lower-doped SL is prepared with two ohmic contact stripes on the sides and voltage pulses up to $38 \mathrm{~V}$ (electric field $\mathrm{F}=190 \mathrm{~V} / \mathrm{cm}$ ) and $10 \mu$ s duration are applied to them with a repetition rate of the order of $100 \mathrm{~Hz}$. The transmission experiment is performed using the Fourier-transform spectrometer in a gated step-scan mode Twhere IR spectra can be recorded during a short time window and the spectrometer is synchronized with the electric field pulses. The sample is held at a lattice temperature of $T_{L}=10 \mathrm{~K}$.

Fig. 5 shows the differential transmission spectra for different electric-field values. The transmission spectra during an electric-field pulse have been recorded and divided by a spectrum taken $10 \mu$ s after the pulse. For comparison with the temperature dependent spectraT the data of Fig. 4 are replotted in Fig. 6. Here the transmission spectra at different temperatures are divided by the spectrum at $T_{L}=10 \mathrm{~K}$. The striking similarity between Figs. 5 and 6 indicates that the assumption of a quasi-thermal electron distribution function is well justified. The electron temperature $\Gamma T_{e} \Gamma$ at each electric field can now unambiguously be determined by comparing both figuresTi.e. Teach electric-field value in Fig. 5 can be assigned to an electron temperature.

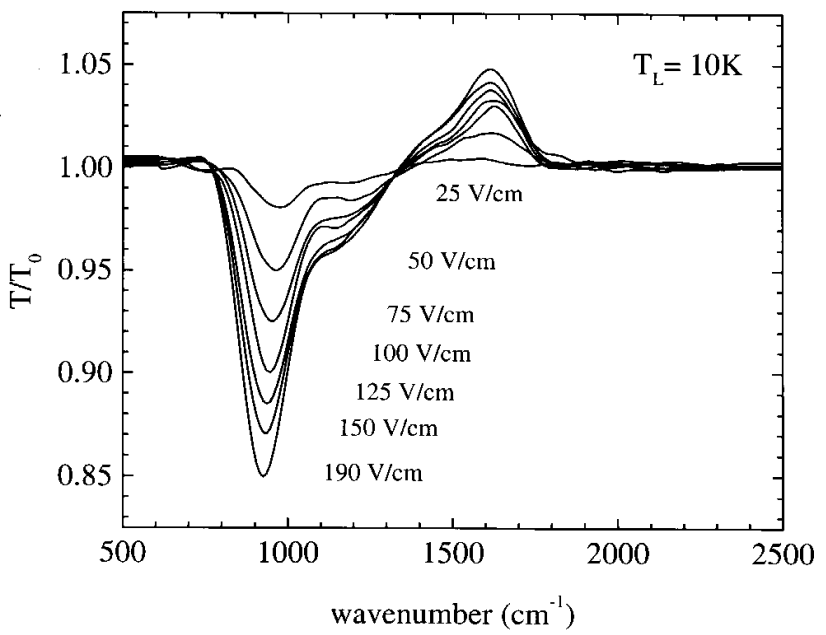

Figure 5. Electric-field dependence of the transmission change: The transmission spectrum at the time of the voltage pulse is divided by the transmission after the pulse. The respective electric- field values are indicated.

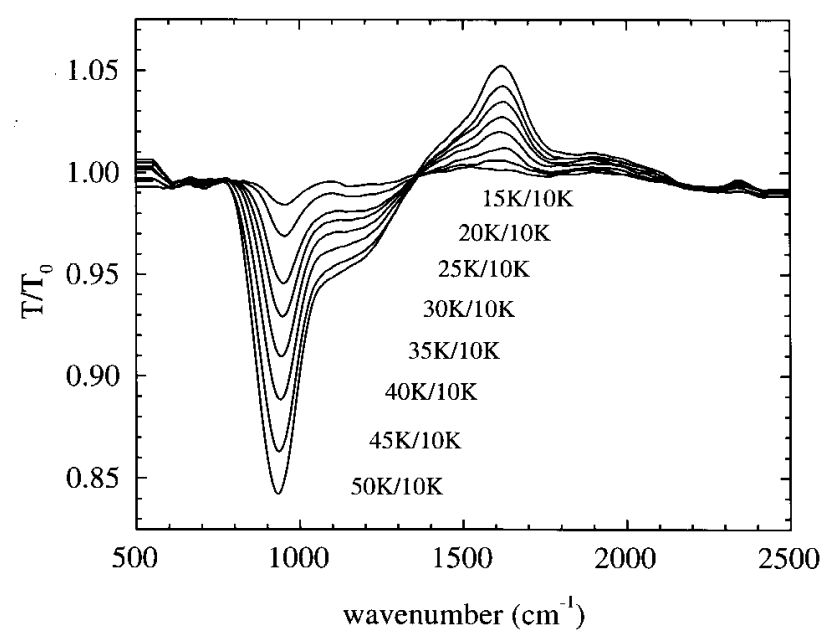

Figure 6. Ratio of the transmission at the lattice temperature $T_{L}$ (as indicated) to the transmission at $T_{L}=10 \mathrm{~K}$.

From these data the average of the power loss and energy relaxation rate of the electron system as a function of electron temperature can be determined in the following way. The power loss per electron is given by $e \mu F^{2}$ Twhere $\mu$ is the electron mobilityltaken from temperature dependent mobility measurements (typically a few $1000 \mathrm{~cm}^{2} / \mathrm{Vs}$ ). Then an average energy relaxation time $\Gamma \tau_{e} \Gamma$ can be determined via the balance equation

$$
e \mu F^{2} \tau_{e}=<\epsilon\left(T_{e}\right)>-<\epsilon\left(T_{L}\right)>.
$$

Here $\left\langle\epsilon\left(T_{e}\right)>\right.$ and $\left\langle\epsilon\left(T_{L}\right)>\right.$ are the carrier energies averaged over the hot distribution function at an electron temperature $T_{e}$ and at equilibrium (where $\left.T_{e}=T_{L}\right)$ Trespectively. We have evaluated the rhs of 
eq. (3) using the numerically calculated SL band structure. The resulting energy relaxation time as a function of electron temperature is shown in Fig. 7. It continuously decreases from $400 \mathrm{ps}$ at $15 \mathrm{~K}$ to about $20 \mathrm{ps}$ at $48 \mathrm{~K}$. A more detailed analysis [5] shows that at low electron temperature the energy loss is governed by emission of acoustic phonons Twhereas at higher temperature $\left(T_{e}>30 \mathrm{~K}\right)$ optical phononsTemitted by electrons in the high-energy tail of the distribution functions $\Gamma$ dominate the relaxation. These values can be compared with a momentum relaxation time of around $0.1 \mathrm{ps}$ as deduced from the mobility Twhich is dominated by impurity scattering.

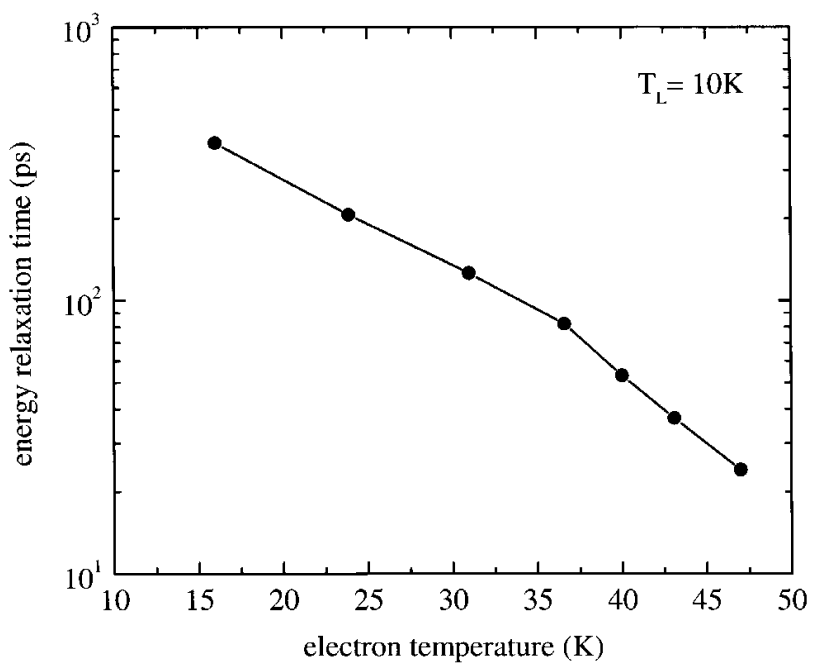

Figure 7. Energy relaxation time as a function of electron temperature.

\section{Vertical transport and Wannier-Stark ladders}

A vertical electric field $\Gamma$ applied perpendicular to the layers of a superlattice $\Gamma$ is known to split up the minibands into a ladder of localized states $\Gamma$ the Wannier Stark ladder (WSL). Its existence has been demonstrated through interband optical experimentsTinvolving transitions from the valence to the conduction band [2]. In a semiclassical picture Tthe electron can reach the inflection point at the edge of the mini-Brillouin zone $\Gamma$ giving rise to negative differential resistance (NDR) in the current-voltage characteristics and to Bloch oscillation [12T13] $\Gamma$ which is the semiclassical analogue of the Wannier-Stark ladder. Bloch oscillations have been observed directly in the time domain by optically exciting electron wavepackets with a sub- picosecond laser pulse [3]. NDRThoweverTalso arises in more weakly coupled superlattices $\Gamma$ where transport proceeds by sequential resonant tunneling $\Gamma$ and is usually accompanied by the formation of electric-field domains [14 115]. In the very high-field regime $\Gamma$ the resonant Zener breakdown has been observed [16] as well as infrared emission from Wannier-Stark ladders [17].

The experimental methods discussed in the previous section can now be employed to study the interminiband absorption under application of a vertical electric field. This opens up the possibility to spectroscopically investigate the above mentioned phenomena in the infrared domainTusing transitions between electron states only. Such type of investigations have only been performed on isolated quantum wells (with thick barriers) $\Gamma$ showing phenomena such as Stark shifts [18] or transfer of oscillator strengths in coupled quantum wells [19]. On the other handTnote that transport and interband optical experiments in a superlattice have been reported [20].

In strongly coupled superlattices $\Gamma$ the finite miniband width gives rise to large current densities in a vertical electric field $\Gamma$ which are of the order of $j \approx 10^{3}$ to $10^{4} \mathrm{~A} / \mathrm{cm}^{2} \Gamma$ corresponding to vertical resistivities $\Gamma$ $V / j \Gamma$ below the $10^{-3} \Omega \mathrm{cm}^{2}$ range. Thus very small mesa structures $(<<100 \mu \mathrm{m}$ diameter) would be required to avoid excessive influence of parasitic series resistances and sample overheating. On the other hand $\Gamma$ the minimum mesa size for infrared measurements is around $500 \mu \mathrm{m}$. In order to get around this problem $\Gamma$ we compromise on the sample design. We design a superlattice with a rather narrow first miniband (to keep the current density reasonably low) $\Gamma$ but a rather wide second miniband $\Gamma$ to achieve still strong coupling of the excited states. The sample is a 300 period $\mathrm{GaAs} / \mathrm{Al}_{0.29} \mathrm{Ga}_{0.71} \mathrm{As}$ superlattice with $50 \AA$ wide quantum wells and $80 \AA$ thick barriersTsandwiched between $n^{+}$GaAs contact layers. The resulting widths of the first and second miniband $\Gamma \Delta_{1}$ and $\Delta_{2}$ Tare $1.2 \mathrm{meV}$ and 30 meV Trespectively. The zero-field band structure is shown in the left inset of Fig. 8 . Note that the second miniband lies in the continuum just above the barriers (right inset of Fig. 8). The superlattice is doped in the central $30 \AA$ of the barriers $\Gamma$ giving an areal electron concentration of $2.25 \times 10^{11} \mathrm{~cm}^{-2}$ per period. 


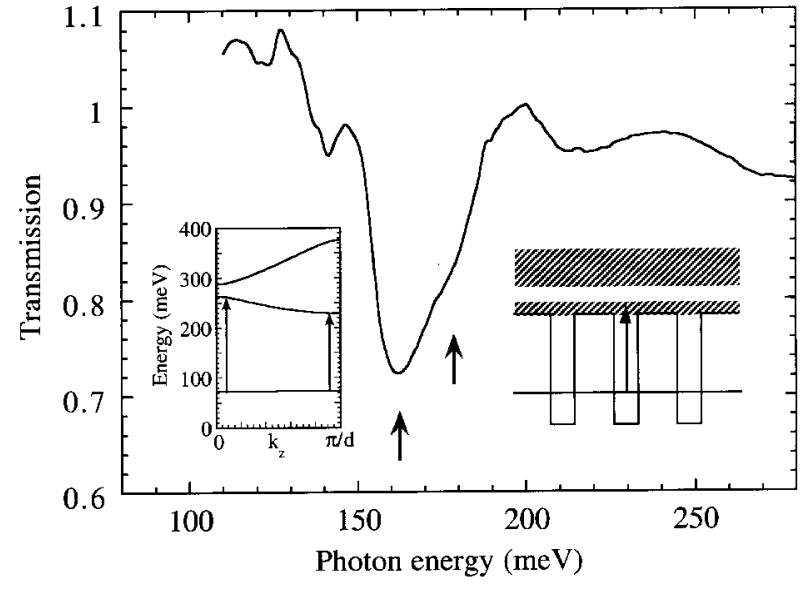

Figure 8. Interminiband transmission spectrum for zero electric field. The insets show the absorption process in k-space and real-space, respectively. The main absorption and the high-energy shoulder, due to the singularities in the joint-density of states, are indicated by arrows.

For the IR absorption measurements $\Gamma 1 \mathrm{~mm}^{2}$ mesa structures were prepared with wedged facets in order to couple the IR light to the intersubband transitions. Fig. 8 shows the zero-field interminiband transmission spectrum of the superlattice Tobtained by ratioing the p- and s-polarization. The main absorption maximum at $164 \mathrm{meV}$ and the high-energy shoulder at $180 \mathrm{meV}$ (both indicated by arrows) are due to the singularities of the joint density of states at $k_{z}=\pi / d$ and $k_{z}=0$, respectively. This is illustrated in the left inset $\Gamma$ which shows the three lowest minibands.

In Fig. 9 the current-voltage characteristic of a 200 $\mu \mathrm{m}$ mesa is shown. At low bias the conduction is ohmic and proceeds by ground-state to ground-state tunneling up to a voltage of $0.5 \mathrm{~V}$ [21]. There the SL breaks up into a low- and a high-field domain; in the latter the electrons tunnel from the ground state in one well to an excited state in a subsequent well (usually the adjacent well). Its extent increases with increasing bias $\Gamma$ until the field distribution is again homogeneous and the current rises steeply. In the present superlattice this occurs at 27 VTi.e. T the voltage drop per period is $27 \mathrm{~V} / 300=90 \mathrm{mV}$. Since this is only about half the value of the subband separation $\Gamma E_{2}-E_{1}=E_{21} \approx 170$ meVTwe conclude that in the high-field domain electrons do not tunnel to the adjacent well एbut rather to the next-nearest SL period $\Gamma$ traversing two barriers and one well (see inset of Fig. 9). This is the first observationTof resonant tunneling over two periods in a regular superlattice [22].

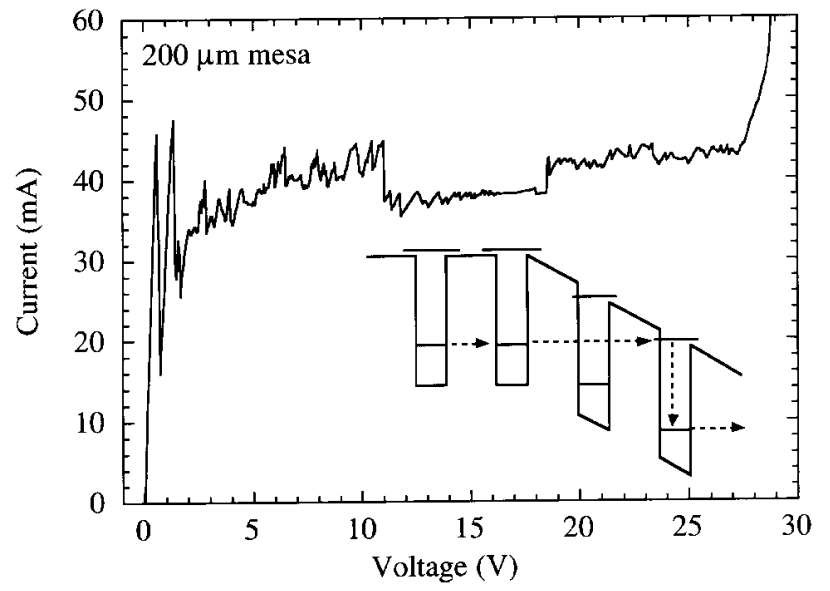

Figure 9. Current-voltage characteristic of the superlattice. The inset shows the conduction-band edge near the boundary of the low- and high-field domain together with a schematic of the transport process.

For the measurement of the IR absorption spectrum in a vertical electric field [6] Tvoltage pulses of about 10 $\mu$ s duration with a repetition rate of a few $100 \mathrm{~Hz}$ were applied to a $1 \mathrm{~mm}^{2}$ superlattice mesa. The transmission change $\Gamma \Delta T=T(F) / T_{0} \Gamma$ is again measured by dividing the signal during the electric-field pulse $\Gamma T(F) \Gamma$ by the signal taken a few ten $\mu$ s after the pulse $\Gamma T_{0}$. Fig. 10 shows a series of traces for increasing voltage. At first note that minima in the signal correspond to decreased transmissionTi.e. they essentially correspond to absorption lines induced by the electric field (marked by arrows). Maxima correspond to frequencies $\Gamma$ where the absorption is reduced compared to the zero-field spectrum. Remarkably the positions of the maxima and minima hardly change with bias Tbut $\Delta T$ continuously increasesTreaching up to $30 \%$ at the highest voltage. This is completely consistent with the observations in the I-V characteristics. Two different electric-field values exist in the sample $\Gamma$ which are realized in two spatial domains; one is close to zero (in the low-field domain) $\Gamma$ the other one (in the high-field domain) is approximately $F=85 \mathrm{mV} / 130 \AA=65 \mathrm{kV} / \mathrm{cm}$. Increasing the voltage only increases the relative length of the high-electric-field domainTresulting in a larger optical signal. Above $30 \mathrm{~V}$ the current increases so steeply that no measurements can be performed in this regime. At very low voltagesTon the other hand Tbelow the onset of domain formationTno transmission change is observed $\Gamma$ since the wavefunction overlap and thus the matrix elements of indirect WS transitions are still too weak. 


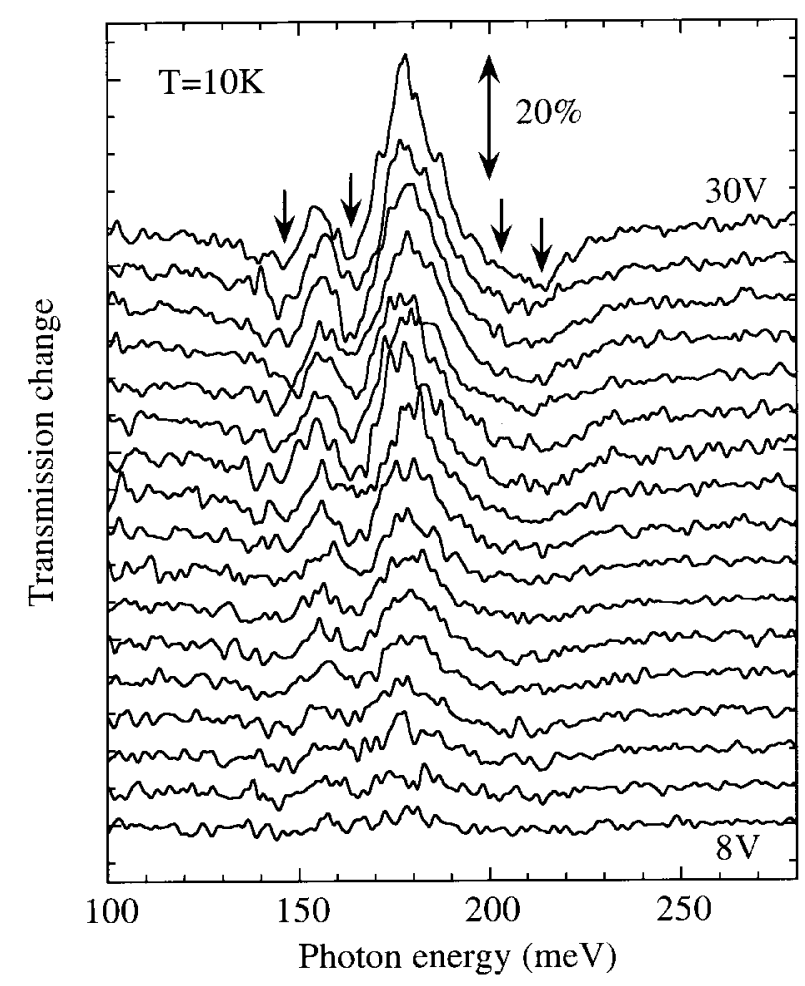

Figure 10. Differential transmission spectrum $\left(T(F) / T_{0}\right)$ of the superlattice for a series of bias voltages between 8 and $30 \mathrm{~V}$. Minima essentially correspond to electric-field-induced absorption lines (marked by arrows).

Understanding the transmission change spectra of Fig. 10 requires a calculation of the absorption coefficient $\Gamma \alpha \Gamma$ under bias. For this purpose we have calculated the energy spectrum $\Gamma$ the wavefunctions and the optical matrix elements between the ground state of one period and all excited statesTusing a finite system of $\mathrm{N}=19 \mathrm{SL}$ periods. The absorption coefficient $\alpha$ is proportional to

$$
\alpha_{n}(\omega)=\text { const. } \times \sum_{n^{\prime}=1}^{N} f_{n n^{\prime}} \frac{\Gamma / \pi}{\left(E_{n^{\prime}}-E_{n}-\hbar \omega\right)^{2}-\Gamma^{2}}
$$

where $n$ indicates the initial state (ground state of one SL period) $\Gamma n^{\prime}$ are the final statesTand $f_{n n^{\prime}}$ is the oscillator strength Tobtained from the optical matrix elements. A Lorentzian broadening $\Gamma=7 \mathrm{meV}$ of each transition is assumed. The calculated absorption coefficient is plot- ted in Fig. 11 for electric fields from $F=0$ up to $F=70$ $\mathrm{kV} / \mathrm{cm}$. Only up to $20 \mathrm{kV} / \mathrm{cm}$ the splitting of the interminiband absorption into a regular Wannier-Stark ladder can be observed. At higher fieldsTstrong mixing of several continuum Wannier-Stark ladders occurs due to Zener coupling [16 [23] and the interpretation of the spectra is far from straightforward. The strong Zener coupling results from the fact that $e F d$ is much larger than the minigaps in the continuum.

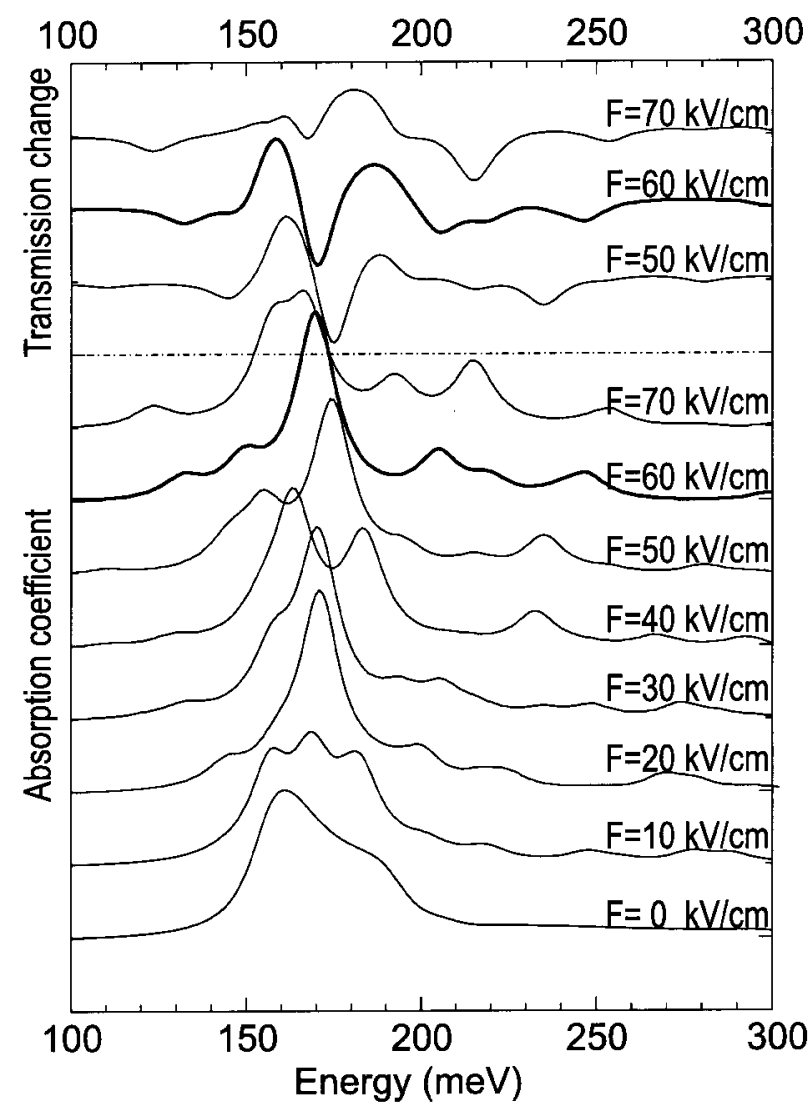

Figure 11. Absorption coefficient calculated for electric fields from 0 to $70 \mathrm{kV} / \mathrm{cm}$. In addition, the transmission change is shown for 50,60 , and $70 \mathrm{kV} / \mathrm{cm}$ in the upper panel to facilitate comparison with the experiment (see text). The experimentally relevant $60 \mathrm{kV} / \mathrm{cm}$ spectra are plotted with thicker lines (compare with Fig. 10).

For direct comparison with experiment $\Gamma$ where $\Delta T=T(F) / T_{0}$ is measured $\Gamma$ we have to calculate the difference $\alpha(0)-\alpha(F)$ rsince

$$
\frac{T(F)}{T(0)}=\frac{\exp (-\alpha(F) d)}{\exp (-\alpha(0) d} \exp [(\alpha(0)-\alpha(F)) d] \cong 1+[\alpha(0)-\alpha(F)] d
$$


for small absorption changes $\Gamma$ as observed experimentally. This is plotted in the top part of Fig. 11 for $F=50 \Gamma 60 \Gamma$ and $70 \mathrm{kV} / \mathrm{cm}$. Comparison with the experimental tracesT Fig. 10Гshows that most of the experimental features can be reasonably well reproduced for a field of $F=60 \mathrm{kV} / \mathrm{cm}$ (drawn by thicker lines for clarity); only the maximum at $\approx 180 \mathrm{meV}$ is larger than predicted $\Gamma$ probably since its strength is very sensitive to the shoulder in the $T_{0}$ spectrum. The above field corresponds to a voltage drop of $80 \mathrm{meV}$ per SL period $\Gamma$ which is very close to half the energy separation $E_{21} \Gamma$ and thus confirms the tunneling to the next-nearest SL period.

In order to obtain a more graphical understanding of the transitions dominating the absorption spectrum $\Gamma$ we attempt a classification of the energy levels in terms of pure WSL quantum numbersTneglecting the interaction due to Zener coupling for the moment. Then the energy spectrum can be written as

$$
E_{m, p}=\epsilon_{m}+p e F d,
$$

where $m=12 \Gamma$. is the (zero-field) miniband indexTand $p=\ldots-1 \Gamma 0 \Gamma 1 \Gamma \ldots$... is the spatial WSL index. Such an assignment can be done by inspecting in detail the wavefunctions and oscillator strengths over several SL periods. Transitions occur from states $\mid m=1, p>$ to $\mid m^{\prime}, p^{\prime}>$ and for brevity $\Gamma$ we term such a transition $\left(m^{\prime}, p^{\prime}-p\right)$; so $\mathrm{Cfor}$ example $(2 \mathrm{~F} 1)$ represents a spatially indirect transition to a WS state localized in the next downhill QW and associated to the second miniband. Fig. 12 shows the resulting assignment graphically. The main part of the relevant wavefunctions are drawn bold and also the calculated absorption coefficient is shown for comparison. The main observed transitions (indicated by arrows) areTfrom low to high energies $\Gamma(3 \mathrm{~F} 1) \Gamma$ (210) (this is the main vertical intersubband transition) $\Gamma$ (3I0) $\Gamma$ and (4F2). Thus $\Gamma$ besides the direct transitions $\Gamma$ we observe indirect transitions up to the next-nearest downhill QW $\left(p^{\prime}-p=-2\right) \Gamma$ and up to states related to the fourth miniband $\left(m^{\prime}=4\right)$. Note that the (4F2) state is as far as $200 \mathrm{meV}$ above the top of the barrier $\Gamma$ when measured at its associated quantum well $\Gamma$ $p^{\prime}-p=-2$. The lowest- and highest-energy transitions (at 132 and $247 \mathrm{meV}$ ) predicted by the calculation are not resolved in the experiment.

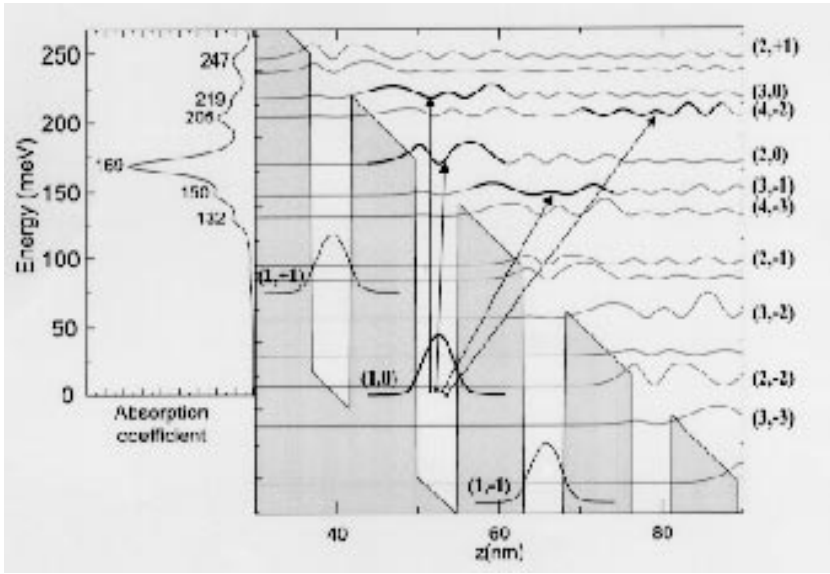

Figure 12. Conduction-band profile, energy levels and squared wavefunctions drawn for four superlattice periods with an electric field of $60 \mathrm{kV} / \mathrm{cm}$. The calculation was performed for a 19 period SL. The experimentally observed transitions are indicated by arrows and the main parts of the respective wavefunctions are emphasized by thicker lines. The numbers on the right represent an (approximate) assignment in terms of WS states (see text). In addition, the calculated absorption coefficient is shown on the left for comparison, including the peak photon energies.

As seen in Fig. 12 Tthe continuum WSL wavefunctions are distinctly different from the usual 5 symmetrically localized tight-binding type wavefunctions [24]. They are very asymmetric and extended far towards the anodeTpartly exhibiting Airy-function character. Note also that due to the finiteness of the structure used in the calculationTno perfect periodicity of the solution for the wavefunctions under a translation $z \rightarrow z+d$ and $E \rightarrow E-e F d$ is obtained. For example $(2 \pi)$ and (2F1) consist of two anticrossed levels 5 whereas $(210)$ of only one level. The absorption coefficient Thowever Twhich is the observable quantityTfulfills this symmetry to a much better degree $\Gamma$ when calculated with a finite broadening $\Gamma$. In other words 5 the oscillator strength per finite energy interval $\Gamma$ is independent of the quantum number $p \Gamma$ despite differences in the wavefunctions. This shows the relation of $\Gamma$ to the coherence length; when $\Gamma$ is very small $\Gamma$ the wavefunctions more easily feel the edges of the finite SL [25].

\section{Summary}

We have attempted to review our investigations on interminiband absorption in superlattices; in particular $\Gamma$ we have discussed the absorption spectrum in terms of the joint-density of states and the oscillator strength. 
With an electric field applied in the SL planesTenergy relaxation times can be determined by employing the temperature dependence of the absorption spectrum. Finally we have described the first infrared absorption measurement on a biased superlattice $\Gamma$ which has evidenced the existence of interpenetrating Wannier-Stark ladders in the continuum of a superlattice. In the future $\Gamma$ similar experiments extended to the far-infrared spectral region may unveil the intra-miniband [26] spectral response (and possibly gain) of a biased superlattice under Bloch-oscillation conditions.

\section{Acknowledgment}

We would like to thank A. Wacker for helpful discussions on superlattice transport and K. Alavi and R. N. Pathak for the growth of two samples. This work was supported by the FWF (Austria) Tby the GMe (Austria) and by the FWO-Vl (Belgium). R.D.M. is supported by IWT (Belgium).

\section{References}

[1] H. T. Grahn (editor), Semiconductor Superlattices (World Scientific, Singapore, 1995).

[2] E. E. Mendez, F. Agullo-Rueda and J. M. Hong, Phys. Rev. Lett. 60, 2426 (1988); P. Voisin, J. Bleuse, C. Bouche, S. Gaillard, C. Alibert and A. Regreny, Phys. Rev. Lett. 61, 1639 (1988).

[3] K. Leo, Semicond. Sci. Technol. 13, 249 (1998) and references therein.

[4] M. Helm, Semicond. Sci. Technol. 10, 557 (1995).

[5] W. Hilber, M. Helm, K. Alavi and R. N. Pathak, Appl. Phys. Lett. 69, 2528 (1996).

[6] M. Helm, W. Hilber, G. Strasser, R. DeMeester, F. M. Peeters and A. Wacker, Phys. Rev. Lett. 82 (1999), in print..

[7] see, e.g. S. L. Chuang: Physics of Optoelectronic Devices, Wiley (New York, 1995), p.373.

[8] B. F. Levine, J. Appl. Phys. 74, R1 (1993).

[9] J. Faist, F. Capasso, D. L. Sivco, C. Sirtori, A. L. Hutchinson and A. Y Cho, Science 264, 553 (1994).
[10] M. Helm, W. Hilber, T. Fromherz, F. M. Peeters, K. Alavi and R. N. Pathak, Phys. Rev. B 48, 1601 (1993).

[11] G. Scamarcio, F. Capasso, C. Sirtori, J. Faist, A. L. Hutchinson, D. L. Sivco and A. Y. Cho, Science 276, $773(1997)$.

[12] L. Esaki and R. Tsu, IBM J. Res. Dev. 14, 61 (1970).

[13] A. Sibille, J. F. Palmier, M. Hadjazi, H. Wang, G. Etemadi and E. Dutisseuil, Superlattices Microstruct. 13, 247 (1993).

[14] H. T. Grahn, H. Schneider and K. von Klitzing, Phys. Rev. B 41, 2890 (1990); S. H. Kwok et al., Phys. Rev. B 51, 9943 (1995).

[15] A. Wacker, in: Theory of Transport Properties of Semiconductor Nanostructures, edited by E. Schöll (Chapman and Hall, London, 1998), p. 321.

[16] A. Sibille, J. F. Palmier and F. Laruelle, Phys. Rev. Lett. 80, 4506 (1998).

[17] G. Scamarcio, F. Capasso, A. L. Hutchinson, D. L. Sivco and A. Y. Cho, Phys. Rev. B 57, R6811 (1998).

[18] A. Harwit and J. S. Harris, Jr., Appl. Phys. Lett. 50, 685 (1987); Y. Mii, R. P. G. Karunasiri, K. L. Wang, M. Chen and P. F. Yuh, Appl. Phys. Lett. 56, 1986 (1990).

[19] J. Faist, F. Capasso, A. L. Hutchinson, L. N. Pfeiffer and K. W. West, Phys. Rev. Lett. 71, 3573 (1993).

[20] A. Sibille, J. F. Palmier and F. Mollot, Appl. Phys. Lett. 60, 457 (1992).

[21] Since in our sample the broadening is somewhat larger than the width of the first miniband, we are rather in the regime of sequential resonant tunneling than of miniband conduction. See also: A. Wacker and A.-P. Jauho, Phys. Rev. Lett. 80, 369 (1998).

[22] Note that tunneling over several periods has been recently observed in superlattices with a basis by: A. Kristensen, P. E. Lindelof, C. B. Sørensen and A. Wacker, Semicond. Sci. Technol. 13, 910 (1998).

[23] H. Schneider, H. T. Grahn, K. v. Klitzing and K. Ploog, Phys. Rev. Lett. 65, 2720 (1990).

[24] J. Bleuse, G. Bastard and P. Voisin, Phys. Rev. Lett. 60, 220 (1988); M. Saitoh, J. Phys. C5, 914 (1972).

[25] C. Rauch, G. Strasser, K. Unterrainer, W. Boxleitner, E. Gornik and A. Wacker, Phys. Rev. Lett. 81, 3495 (1998).

[26] G. Brozak, M. Helm, F. DeRosa, C. H. Perry, M. Koza, R. Bhat and S. J. Allen, Jr., Phys. Rev. Lett. 64, 3163 (1990). 